\title{
Broadband biphotons in a single spatial mode
}

\author{
K. G. Katamadze, ${ }^{1,2, *}$ N. A. Borshchevskaya, ${ }^{1}$ I. V. Dyakonov, ${ }^{1}$ A. V. Paterova, ${ }^{1}$ and S. P. Kulik ${ }^{1}$
}

${ }^{1}$ M. V. Lomonosov Moscow State University, 119992, Moscow, Russia

${ }^{2}$ Institute of Physics and Technology, Russian Academy of Sciences, 117218, Moscow, Russia (Dated: January 12, 2021)

We demonastrate the experimental technique for generating a spatial single-mode broadband biphoton field. The method is based on a dispersive optical element which precisely tailors the structure of the type-I SPDC frequency angular spectrum in order to shift different spectral components to a single angular mode. Spatial mode filtering is realized by coupling biphotons into a single-mode optical fiber.

* k.g.katamadze@gmail.com 


\section{INTRODUCTION}

Progress in preparation and manipulation of quantum states of light is boosted by development of quantum communication and quantum computation. Spontaneous parametric down-conversion (SPDC) [1] is the most efficient and widespread source of correlated photon pairs (biphotons). Phenomenologically, one may describe the SPDC process as a spontaneous decay of pump photon $p$ traveling through a $\chi^{(2)}$ nonlinear medium into a pair of photons conventionally referred to as the signal $s$ and idler $i$. Emerged photon pairs obey the energy conservation law and phase matching relation:

$$
\begin{aligned}
& \omega_{p}=\omega_{s}+\omega_{i}, \\
& \vec{k}_{p}=\vec{k}_{s}+\vec{k}_{i}+\vec{\Delta} .
\end{aligned}
$$

Here, $\omega_{p, s, i}$ refer to the pump, signal and idler frequencies respectively, $\vec{k}_{p, s, i}$ - corresponding wave vectors, and $\vec{\Delta}$ is a phase mismatch responsible for the decay probability [1]. In the present work we will concentrate on investigating the frequency and angular characteristics of biphotons generated in the SPDC process. A biphoton quantum state may be expressed as

$$
|\Psi\rangle=F\left(\omega_{s}, \theta_{s}, \omega_{i}, \theta_{i}\right) a_{s}^{\dagger}\left(\omega_{s}, \theta_{s}\right) a_{i}^{\dagger}\left(\omega_{i}, \theta_{i}\right)|v a c\rangle,
$$

where $a_{s}^{\dagger}\left(\omega_{s}, \theta_{s}\right)$ and $a_{i}^{\dagger}\left(\omega_{i}, \theta_{i}\right)$ represent photon creation operators in frequency modes $\omega_{s, i}$ and angular modes $\theta_{s, i}$, and $F\left(\omega_{s}, \theta_{s}, \omega_{i}, \theta_{i}\right)$ is a spectral amplitude of the biphoton. Relation between angular and frequency modes of a pair in monochromatic plane-wave pump approximation is given by:

$$
n\left(\omega_{i}\right) \omega_{i} \sin \theta_{i}=n\left(\omega_{s}\right) \omega_{s} \sin \theta_{s},
$$

where $n(\omega)$ is refractive index. Hence, the two-photon spectral amplitude $F\left(\omega_{s}, \theta_{s}, \omega_{i}, \theta_{i}\right)$ reduces to a function of two variables $\tilde{F}\left(\omega_{s}, \theta_{s}\right)$. The typical X-shape of the frequency-angular spectrum of the biphoton field $\left|\tilde{F}\left(\omega_{s}, \theta_{s}\right)\right|^{2}$ generated under collinear degenerate type-I phase-matching conditions is depicted in Fig 1 a. For applications purposes a spatial single-mode biphoton field generation regime is preferential. A pump beam waist, which is typically $W \gtrsim 50 \mu \mathrm{m}$, defines photon pair emission cone $\Delta \theta=\frac{2 \lambda}{\pi W}$. This relation implies a narrow angular emission range in the single-mode generation regime.

Spectral broadening of the single spatial mode biphoton field remains a really significant puzzle among a wide list of biphoton spectrum control problems. The broadband biphotons exhibit ultra-short temporal correlations [1] 3]. Their correlation time $\Delta \tau \sim 1 / \Delta \nu$. Ultracorrelated biphotons can be utilized in metrological applications such as distant clock synchronization [4, quantum optical coherence tomography (QOCT) [5, 6] and quantum interferometric optical lithography [7, 8].

Besides, spectral broadening increases the degree of biphoton entanglement [9, which can be exploited in quantum communication tasks. A quantum information can be directly encoded in frequency bins [10, 11] or the broad spectrum can be used for a wavelength-multiplexed entanglement distribution [12 16].

Also biphoton spectrum broadening increases a maximum pair generation rate $R_{\max } \sim \Delta \nu$ [17] enabling an extremely high-speed quantum communication.

Typically a biphoton spectral bandwidth is limited by an interaction medium dispersion $\Delta(\omega)<2 \pi / L$, where $L$ is an interaction length. Hence, additional biphoton spectral broadening methods should be developed. Homogeneous spectral broadening can be achieved by using the special nonlinear media with zero dispersion [18 21] $(\Delta \tau=5$ fs [19] and $\Delta \nu=100 \mathrm{THz}$ [20] were obtained for type-I SPDC). Reducing nonlinear interaction length, one can generate $100 \mathrm{THz}$ bandwidth type-II SPDC [22] and $150 \mathrm{THz}$ type-I [23], but this method essentially decreases an intensity of biphoton radiation. Pump beam focusing [24 26] and pump spectrum broadening [27, 28] also enable the broadband biphoton generation with a bandwidth up to $83 \mathrm{THz}$ [28]. One more method of homogeneous SPDC broadening is based on the modifying a crystal's effective dispersion by means of a pump pulsefront tilt combined with a spatial walk-off [29]. A $44 \mathrm{THz}$ bandwidth type-II SPDC spectrum was measured and a $197 \mathrm{THz}$ bandwidth $(\Delta \tau=6.4 \mathrm{fs})$ spectrum was theoretically predicted for type-I SPDC.

Biphoton generation in inhomogeneous nonlinear media (a set of crystals [30], chirped crystals [5, 31], crystals with a spatially modulated refractive index [32 34]) leads to inhomogeneous spectral broadening. Up to $154 \mathrm{THz}$ bandwidth of the SPDC spectrum was obtained [34, but such inhomogeneous broadening inevitably leads to a Fourier-unlimited field.

All listed methods aim to increase parameter range (an area on the $\omega-\theta$ coordinate plane) satisfying the phasematching conditions which inevitably leads to a proportional drop in the spectral intensity. The typical view of the frequency-angular spectrum for broadened (namely by reducing a crystal length [23]) type-I SPDC is shown in Fig 1 b.

It was demonstrated in [2, 35] that the up-conversion process evinces an ultra-short correlation time $(\Delta \tau=$ $4.4 \mathrm{fs}$ ) provided that biphotons were emitted into a wide angular range and then refocused onto the second nonlinear medium. However, collecting a wide angular range $\Delta \theta$ results in essentially a multimode biphoton generation regime, which is highly undesirable for long-distance (fiber or free-space) optical communication, microscopy 


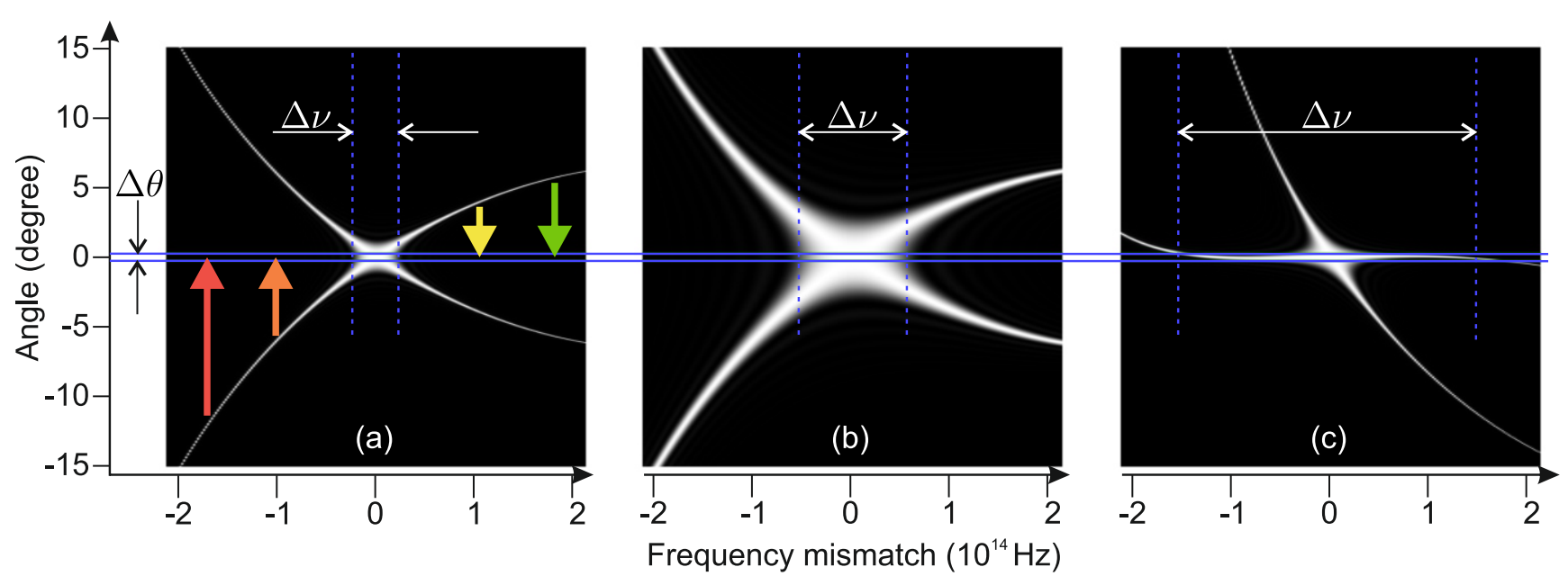

Figure 1. (Color online) The type-I collinear degenerate frequency-angular SPDC spectra, calculated for the $2 \mathrm{~mm}$ BBO crystal (zero on the frequency scale corresponds to the central frequency $\nu_{o}=\omega_{p} / 4 \pi$ ). (a) The initial spectrum. (b) The typical view of the spectrum for usual broadening techniques. (c) The spectrum, transformed by an angular dispersion. The frequency bandwidth $\Delta \nu$ corresponds to the collection angular range $\Delta \theta$.

and interferometric applications. Hence, the spatial single-mode broadband biphoton generation technique is a subject of endeavor for high-dimensional quantum-state engineering problems.

We have to note that the spatial single-mode biphoton generation problem (leaving spectral properties aside) has been thoroughly studied (for example [36-39]). Generally, the solution is reduced to optimization of biphoton coupling conditions to a single-mode fiber. This implies maximization of single photon and coincidence counting rates and their ratio (the single to coincidence counting rate ratio is a key parameter in terms of pure singlephoton state preparation). Our efforts were solely concentrated on achieving the maximal spectral width of a spatial single-mode biphoton source.

\section{METHOD DESCRIPTION}

In spite of the fact that the total biphoton spectrum is broad both in frequency and angle, there is one-toone correspondence between angles and frequencies due to phasematching. We propose to reconfigure the SPDC spectrum by means of an angular dispersive optical element, shifting different spectral components to a single angular mode (Fig. 1 c). This method doesn't decrease the spectral intensity of SPDC radiation and significantly increases the integral intensity in the target angular mode.

The principal scheme of the experimental setup is depicted in Fig. 2. Different biphoton spectral components propagate in different angular modes. The lens system focuses emission onto an angular dispersive element (diffraction grating). Such a system transforms the SPDC angular spectrum to provide congruence with the

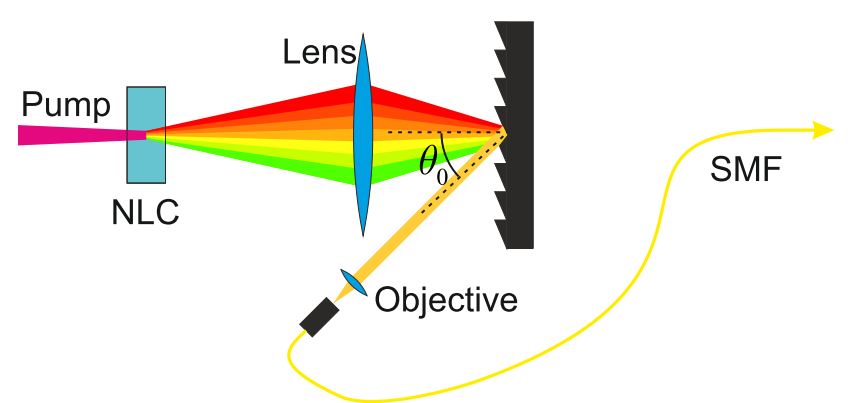

Figure 2. (Color online) The principal scheme of the experimental setup

grating dispersion curve. After diffracting from the grating, the majority of spectral components correspond to the same angular modes and the radiation is coupled by the objective to a single-mode fiber.

We have to point out that an analogous method was previously studied for optical parametric amplifier bandwidth broadening (for example [40 44]). However, it has never been utilized in biphoton generation applications. Nevertheless, biphoton spectral broadening using an angular dispersion module was demonstrated in [29], where the pump pulse-front tilt was implemented by means of a diffraction grating.

\section{MODEL}

The numerical simulation of the SPDC process was evaluated based on the theoretical approach elaborated in 38. We consider only the photon pairs generated in given gaussian signal and idler modes, defined by a 


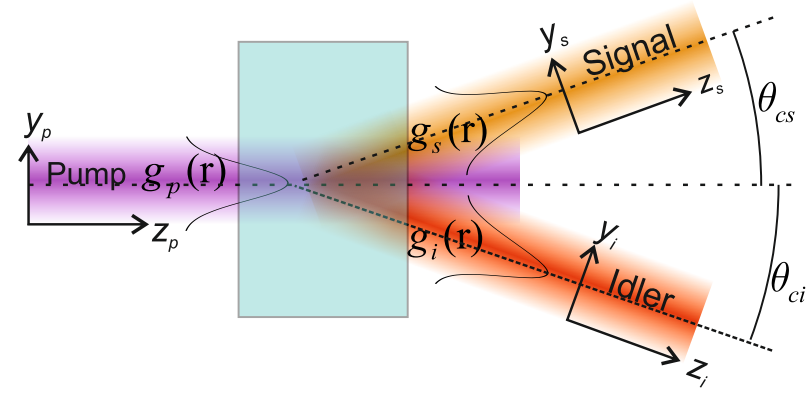

Figure 3. (Color online) The pump, signal and idler gaussian modes.

registration scheme:

$$
E_{j}(\boldsymbol{r}) \sim g_{j}(\boldsymbol{r})=\exp \left(i k_{j} z_{j}\right) \exp \left(\frac{-\left(x^{2}+y^{2}\right)}{W_{j}^{2}}\right),
$$

where $j=i, s, p$. The pump field $g_{p}$ transverse profile is also assumed to be gaussian (Fig. 3). On the one hand the biphoton emission rate

$$
R_{T} \propto\left|F\left(\omega_{c s}, \omega_{c i}, \delta \omega, \theta_{c s}, \theta_{c i}, W_{s}, W_{i}, W_{p}\right)\right|^{2},
$$

depends on the mode overlap integral, and on the phasematching conditions on the other. Here $\theta_{c s}, \theta_{c i}$ are the central angles of signal and idler beams, $W_{s}, W_{i}$ and $W_{p}$ are corresponding beam waists. The parameter $\delta \omega$ corresponds to spectral bandwidth of detection scheme.

The diffraction grating dispersion relation yields the expression for binding angles $\theta_{c s, i}$ and frequencies $\omega_{c s, i}$ :

$$
\sin \theta_{0}-\sin \gamma \theta_{c s, i}=\frac{2 \pi c D}{\omega_{c s, i}}
$$

where $\theta_{0}$ is the incident angle of a gaussian mode conjugated with the single-mode fiber fundamental mode (Fig. 2), $D$ is the diffraction grating groove density and $\gamma$ is the lens system magnification. The signal and idler beam waists $W_{s, i}$ are related with the wavelengths $\lambda_{s, i}$ through

$$
W_{s, i}=\Gamma W_{f}\left(\lambda_{s, i}\right),
$$

where $\Gamma$ is the lens and condensing objective optical system magnification and $W_{f}$ is the fiber fundamental mode beam waist, which is wavelength dependent:

$$
W_{f}=\frac{\lambda}{\pi \mathrm{N} . \mathrm{A} .} .
$$

Here N.A. is the value of the fiber numerical aperture.

Substituting expressions (1), (7)- 9 ) to (6) one can obtain the frequency dependence of biphoton emission rate $R_{T}\left(\omega_{c s}\right)$ governed by experimentally tuned parameters: $\gamma, \Gamma$ and $W_{p}$.

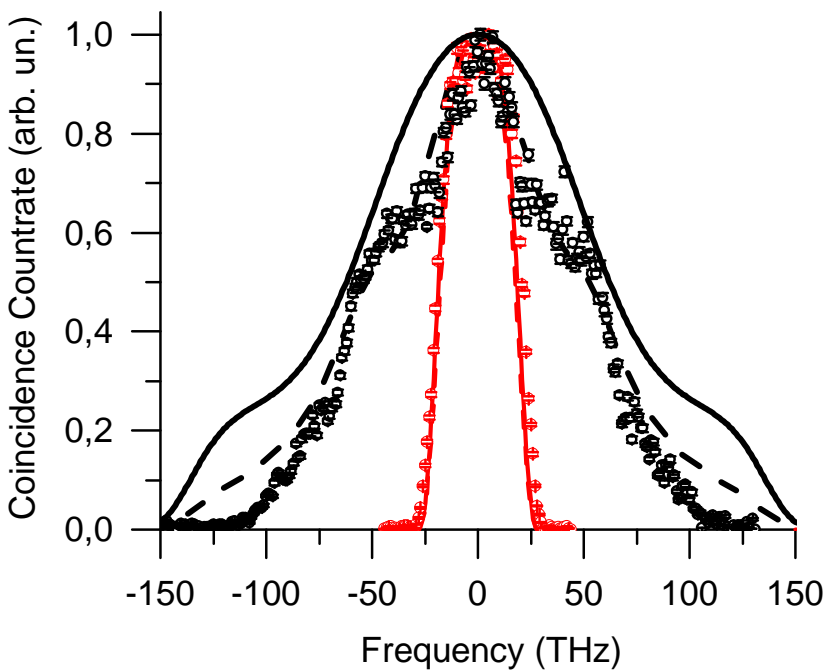

Figure 4. (Color online) Biphoton frequency spectra. The value $\nu=0$ corresponds to the central frequency $\omega_{p} / 4 \pi$. Solid lines correspond to ideal calculation, dashed line corresponds to exact calculation,taking into account the diffraction efficiency, circles correspond to experimental data. Black thick lines and circles refer to transformed spectrum and red thin to initial one.

\section{CALCULATION RESULTS}

Numerical calculations were performed for the nonlinear $2 \mathrm{~mm}$ long BBO crystal cut for a collinear degenerate type-I phasematching, pumped by the $325 \mathrm{~nm}$ laser beam with a waist $W_{p}=34 \mu \mathrm{m}$. Optimal SPDC coupling conditions demand shrinking the pump beam waist to smaller values but, taking into account that for given nonlinear media spatial walk-off is equal to $50 \mu \mathrm{m}$, further pump beam waist reduction won't enhance a SPDC to single-mode fiber coupling efficiency. The diffraction grating groove density was chosen as $600 / \mathrm{mm}$ leading to $\gamma=1.05$ for optimal overlap between the phasematching curve and the diffraction grating dispersion curve. According to [38], to achieve the maximum collecting efficiency $\Gamma$ has to be selected in order to satisfy the condition

$$
W_{s}=W_{i}=\sqrt{2} W_{p}=48 \mu \mathrm{m},
$$

which corresponds to the angular bandwidth $\Delta \theta=0.5^{\circ}$ in Fig. 1. Considering the spectral dependence of $W_{s, i}$, $\Gamma$ was selected to satisfy 10 only for the central wavelength $(650 \mathrm{~nm})$. Initial and transformed calculated coincidence spectra are plotted in Fig. 4 (straight curve).

We used a diffraction grating blazed at $750 \mathrm{~nm}$ in our experiment. The first order diffraction conversion coefficient at $750 \mathrm{~nm}$ was equal to $70 \%$ and drastically dropped down to $25 \%$ at $500 \mathrm{~nm}$. We introduced diffraction efficiency to our numerical model; the result is plotted by 


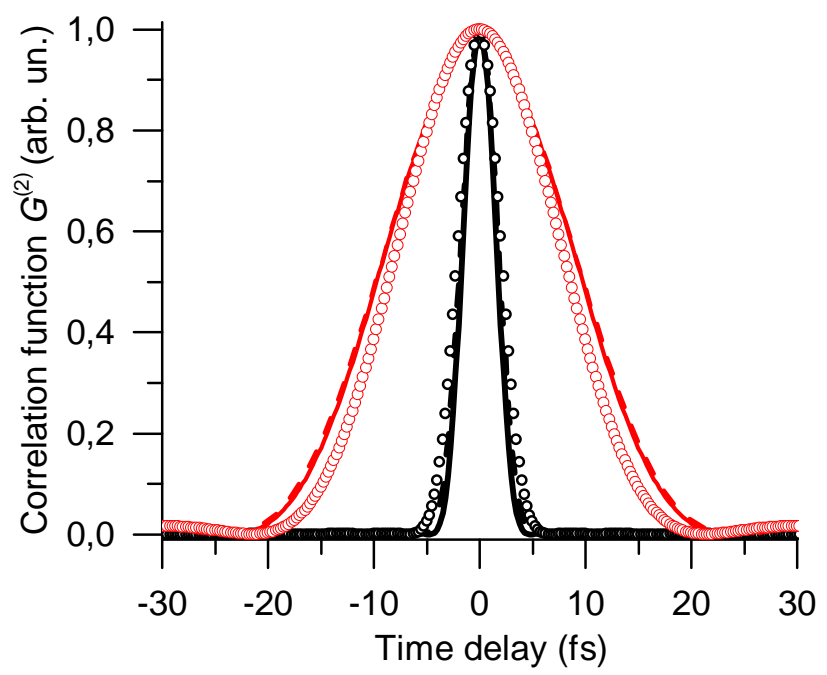

Figure 5. (Color online) Biphoton correlation functions $G^{(2)}$ 12. calculated from the spectra plotted in Fig. 4 Solid lines correspond to ideal calculation, dashed line corresponds to exact calculation,taking into account the diffraction efficiency, circles correspond to experimental data. Black thick lines and circles refer to transformed spectrum and red thin to initial one.

Table I. The spectral bandwidth $\Delta \nu \sqrt{11}$ and the second order correlation time $\Delta \tau$ for the plots in Fig. 4 and Fig. 5

\begin{tabular}{l|cc|ccc}
\hline \hline & \multicolumn{2}{|c|}{ Initial } & \multicolumn{3}{|c}{ Transformed } \\
& Theory & Exp. & \multicolumn{2}{|c}{ Theory } & Exp. \\
& & & Ideal & Exact & \\
\hline$\Delta \nu(\mathrm{THz})$ & 36 & 39 & 144 & 112 & 99 \\
$\Delta \tau(\mathrm{fs})$ & 20.3 & 18.2 & 3.7 & 4.1 & 4.8 \\
\hline \hline
\end{tabular}

a dashed curve in Fig. 4 The values of the spectral bandwidths, determined as an integral characteristic:

$$
\Delta \nu=\int R(\nu) d \nu / R(0),
$$

are presented in Table I. One may note that spectral dependence of diffraction efficiency leads to narrowing of the bandwidth of transformed spectrum from 144 to $112 \mathrm{THz}$.

Next we evaluated the corresponding second order correlation functions

$$
G^{(2)}(\tau) \propto\left|\int F(\omega) e^{-i \omega \tau} d \omega\right|^{2}
$$

which are presented in Fig. 5 The corresponding correlation times are shown in Table [1. The correlation times have been calculated similarly to (11). Ideally, $3.7 \mathrm{fs}$ correlation time can be achieved. It corresponds to 1.7 optical cycles. Non-uniform grating spectral efficiency leads to longer $4.1 \mathrm{fs}$ correlation time.

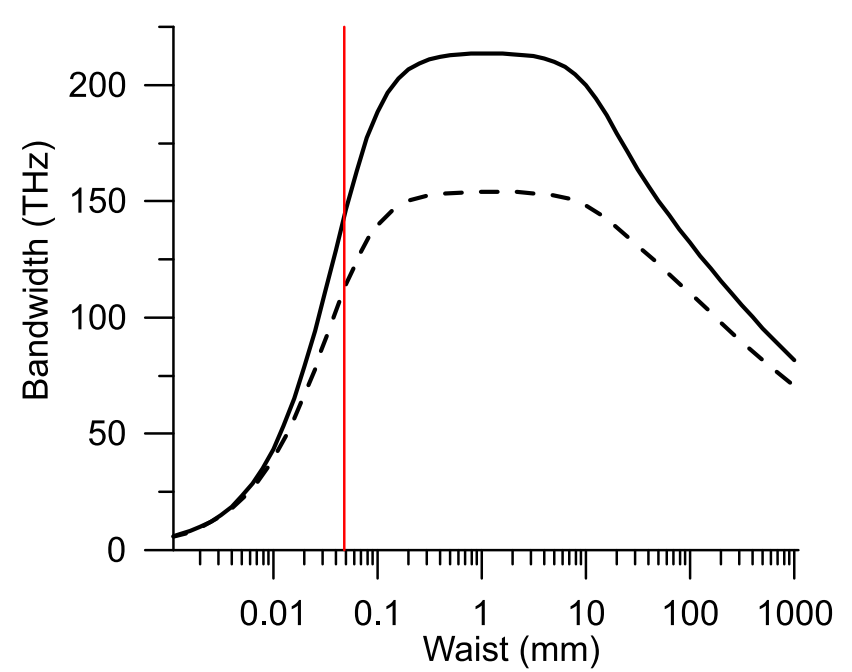

Figure 6. (Color online) The frequency bandwidth 11) dependence on the beam waist. Straight line - the ideal case, dashed line - taking into account the spectral dependence of the grating efficiency. Vertical line - the waist value $W=48 \mu \mathrm{m}$ used in the calculations and in the experiment.

We calculated the spectral bandwidth 11 with respect to SPDC beam waists $W_{s, i}(650 \mathrm{~nm})$ considering the relation for the pump beam waist $(10)$. The results are plotted in Fig. 6. The vertical line corresponds to $W_{s, i}(650 \mathrm{~nm})=48 \mu \mathrm{m}$, included in our numerical model. Evidently, this value appears to be non-optimal considering maximum spectral broadening. Increasing the beam waist up to $500 \mu \mathrm{m}$ leads to broadening of the spectral bandwidth up to $213 \mathrm{THz}$ in an ideal case and up to $154 \mathrm{THz}$ taking into account the diffraction efficiency. On the other hand, larger beam waists imply a significant drop in coincidence count rate. According to 38 the coincidence count rate scales as $\propto W^{-2}$.

For beam waists smaller than $100 \mu \mathrm{m}$ our numerical model predicts a decrease in the spectral bandwidth of the coincidence count rate. This effect is associated with shrinking the interaction volume for a noncollinear generation regime in a nonlinear medium between the pump, signal and idler gaussian beams, which leads to depletion of the spectral intensity of distant spectral components. At the same time, the collection angular range increases allowing the vertical branch of the angular-frequency spectrum (Fig. 1 c) to enhance the central spectral components and, hence, reduce the spectral width. Here we have to note that our model doesn't apply to a tight-focus scenario, when the waist length is shorter than the crystal length. Moreover, it doesn't account for broadening of the SPDC spectrum due to pump focusing [24 26].

Spectrum bandwidth reduction due to the beam waist decreasing is associated with angular range $\Delta \theta$ narrowing, where the phasematching curve overlaps the grating dispersion curve. Hence, the more precise overlap can be 
achieved for the narrower spectral range.

\section{EXPERIMENT}

Fig. 7illustrates the scheme of the experimental setup. Laser radiation at $325 \mathrm{~nm}$ generated by the $\mathrm{HeCd}$ laser was focused by a lens $\mathrm{L} 0\left(f_{0}=145 \mathrm{~mm}\right)$ on the $2 \mathrm{~mm}$ long $\mathrm{BBO}$ crystal cut for collinear degenerate type I phasematching. A dispersive prism and filter F1 blocked parasite arc radiation. The pump beam was blocked with a UV Filter F2. Biphotons were focused through a doublelens system L1 and L2 $\left(f_{1}=f_{2}=40 \mathrm{~mm}\right)$ on the diffraction grating, described in the previous section. The lens positions (noted in Fig. 7] in $\mathrm{mm}$ ) were selected to set the magnification of the system to $\gamma=1.05$. The numerical aperture of the lens system exceeded 0.17 which corresponds to $\pm 9.5^{\circ}$ angular range. It corresponds to the spectral mismatch interval from -150 to $460 \mathrm{THz}$ for the given the phasematching curve (Fig. 1 a). To measure an initial spectrum, the diffraction grating was replaced by a broadband mirror $\mathrm{M}$.

We used a 20X objective lens O1 to couple first-order diffracted light to a single-mode fiber SMF (Thorlabs SM600). This fiber retains single-mode propagation in the $600-800 \mathrm{~nm}$ band. For shorter wavelengths of $500-$ $600 \mathrm{~nm}$ the fiber maintains a few-mode lossless propagation, which may affect the coupling efficiency in the specified spectral range. For our scheme the effect of a non-single-mode coupling is negligible. However, for the near-infrared range starting from $800 \mathrm{~nm}$ the propagation losses become significant. That is why we only used a $1 \mathrm{~m}$ long fiber patch-cord to minimize propagation losses.

Single-mode fiber output radiation was collimated by the objective $\mathrm{O} 2$ and split by a 50:50 nonpolarizing broadband beam splitter BS and coupled with objectives $\mathrm{O} 3$ and $\mathrm{O} 4$ to multimode fibers MMF1 and MMF2 connected with Single-photon counting modules D1 and D2 based on $\mathrm{Si}$ avalanche photodiodes. One of the channels after the beam splitter included a spectrograph prism block, capable of $\delta \nu=2 \div 4 \mathrm{THz}$ (wavelength dependent) spectral band filtering. Sufficiently wide spectral filtering enables both high coincidence count rate and sufficient spectral resolution.

Experimental data took into account all spectral dependent parameters of the measurement part: detector quantum efficiency and wavelength dependent spectral filtering bandwidth. Thus, the key non-ideality of the preparation part (grating efficiency) was taken into account in the above theoretical model.

\section{EXPERIMENTAL RESULTS}

The measured transformed coincidence spectrum is plotted with thick (black) circles in Fig,4. Thin (red)
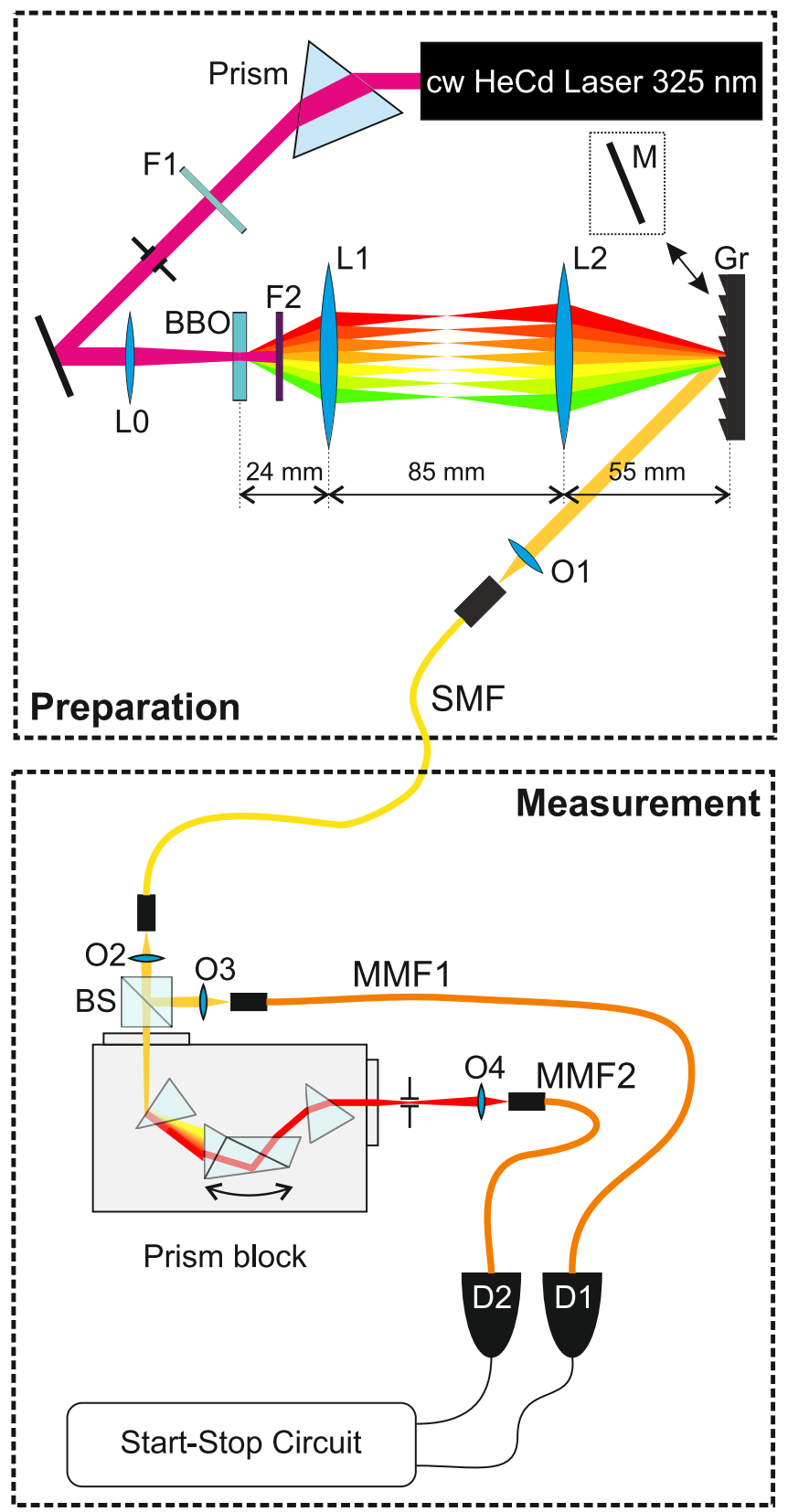

Figure 7. (Color online) The scheme of the experimental setup.

circles refer to the initial spectrum measured after placing the broadband mirror instead of the diffraction grating. All the plots are normalized against their maximal values. The measured spectral intensities at the central frequency for initial and transformed spectra were different, which can be explained by the distinction between the grating efficiency and mirror reflection coefficient.

Agreement between the experimental data and numerical simulation (accounting for diffraction grating efficiency) is observed. The $99 \mathrm{THz}$ spectral bandwidth (11) was obtained. The signal drop on the edges of 
the spectral range is attributed to geometric lens aberrations. Notably, the measured initial spectrum looks slightly broader than the calculated one. It can be explained by the small broadening due to pump focusing [24 26], which wasn't taken into account in the theory.

The second-order correlation functions 12 calculated from the experimental data are also plotted with circles in Fig. 5. The correlation time related to the transformed spectrum is $4.8 \mathrm{fs}$. All spectral bandwidths $\Delta \nu$ and correlation times $\delta \tau$ are presented in Table 1 in the "Exp." columns.

The impact of the single-photon impurity is quantified by the coincidence to single count rates ratio: $1-R c / R s$. We experimentally estimated the ratio $R c / R s$ (at the central frequency) to be $2.5 \%$. The low biphoton intake is attributed to significant optical losses in the measurement part of the setup and an inefficient fiber coupling scheme. This is a trade-off between the maximal broadening and maximal efficiency. Thus, the maximum possible value of $R c / R s$ is $45 \pm 5 \%$ assuming any other losses are negligible. The preparation part of the setup solely introduces optical losses reducing the $R s / R c$ ratio down to $17 \pm 2 \%$.

We measured coincidence count rates $R_{i n}=33 \mathrm{~Hz}$ and $R_{t r}=19 \mathrm{~Hz}$ at the central frequency $(\delta \nu=3.2 \mathrm{THz})$ for initial and transformed biphoton fields to ensure the biphoton spectral power density invariance. The ideal scenario implies $R_{t r} / R_{i n}=1$. The measured ratio $R_{t r} / R_{i n}=0.58$ may be attributed to a significant difference between the diffraction grating efficiency and the broadband mirror reflectance at the given frequency. Moreover, a discrepancy between the measured value of $R_{t r} / R_{i n}$ and the corresponding broadening factor $\Delta \nu_{i n} / \Delta \nu_{t r}=0.39$ endorses the fact that in our experiment the spectral intensity drop isn't induced by biphoton spectral broadening.

\section{DISCUSSION}

The demonstrated biphoton source engineering technique possesses several distinctive advantages. First, the method provides an ultra-broadband biphoton generation regime. The measured $100 \mathrm{THz}$ bandwidth and $5 \mathrm{fs}$ correlation time corresponds to values enabled by the homogeneous broadening techniques [19, 20, 28]. The optimization of our scheme enables bandwidth up to $144 \mathrm{THz}$, which have been demonstrated by using the inhomogeneous [34] or extra-thin [23] nonlinear crystals.

On the other hand, our method is limited by the spectral region, where phasematching and grating dispersion curves coincide, whereas other spectral broadening methods, based on utilizing inhomogeneous nonlinear media [26, 30 34], are completely free of this limitation. At the same time, the biphoton field, generated in inhomogeneous nonlinear media, cannot be kept Fourier-limited, and additional compression techniques are required [17, 45, 46]. The technique, demonstrated in our experiment, provides a Fourier-limited broadband biphoton source. Additional dispersive broadening of the correlation function may arise after passing through the lens system and single-mode fiber [3]. This effect can be avoided by substituting lenses with parabolic mirrors and realising the spatial mode filtering with a pinhole. Thus, our method (reversed in time) can also be applied for precise measurement of second-order correlation time by exploiting the sum-frequency generation scheme. The resolution of the such scheme is limited by the phasematching bandwidth in the nonlinear crystal.

However, our method exhibits significant optical losses, which leads to a considerable additional single-photon component in the state (3). The fact that the fabrication of efficient broadband diffraction gratings is barely feasible makes these kinds of losses inevitable. On the other hand, the losses emanate from non-optimal coupling biphotons to a single-mode fiber. The maximal coupling efficiency requires each biphoton spectral component to populate a single Gaussian mode. It can be realized by irradiating a nonlinear medium with a tightly focused pump beam. In our case the above condition is impossible to achieve for all frequency-angular components at the same time.

It should be noted that the demonstrated method enables broadband biphoton generation in a single spatial mode, whereas applications based on correlation function measurements demand photons of a pair to populate distinct spatial modes. This problem can be solved by a combination of two coherent single-mode biphoton sources [47.

Finally, the demonstrated technique significantly broadens the biphoton frequency spectrum without depleting the spectral intensity (and, moreover, with enhancing the total intensity) by redistributing energy between spatial biphoton modes.

\section{CONCLUSION}

We proposed the novel method of generating a spatial single-mode ultra-broadband biphoton field based on the frequency-angular spectrum transformation by means of the angular dispersion. The efficiency of this method was demonstrated both theoretically and experimentally and our calculations are in a significant agreement with our experimental results. The spectral bandwidth $\Delta \nu>100 \mathrm{THz}$ and the correlation time $\Delta \tau<5$ fs can be reached. The key feature of our method - spectral intensity invariance - was also demonstrated.

\section{ACKNOWLEDGMENTS}

The authors are grateful to Stanislav Straupe for helpful discussions on biphoton spatial modes. 
This work was supported by Russian Foundation of Basic Research (projects 14-01-00557-a, 14-02-00749, and 14-02-31106-mol_a) and in part by the European Union Seventh Framework Programme under grant agreement no. 308803 (project BRISQ2) and NATO Project EAP.SFPP 984397. N. Borshchevskaya is grateful for a support to Russian Science Foundation (project 14-1201338).
[1] A. V. Belinsky and D. N. Klyshko, Laser Physics 4, 663 (1994)

[2] L. Caspani, E. Brambilla, and A. Gatti, Physical Review A 81, 1 (2010)

[3] M. V. Chekhova, JETP Letters 75, 225 (2002).

[4] A. Valencia, G. Scarcelli, and Y. Shih, Applied Physics Letters 85, 2655 (2004).

[5] S. Carrasco, J. P. Torres, L. Torner, A. Sergienko, B. E. a. Saleh, and M. C. Teich, Optics Letters 29, 2429 (2004)

[6] M. Nasr, B. Saleh, A. Sergienko, and M. Teich, Physical Review Letters 91, 083601 (2003).

[7] A. Boto, P. Kok, D. Abrams, S. Braunstein, C. Williams, and J. Dowling, Physical Review Letters 85, 2733 (2000)

[8] M. D'Angelo, M. Chekhova, and Y. Shih, Physical Review Letters 87, 013602 (2001)

[9] G. Brida, V. Caricato, M. V. Fedorov, M. Genovese, M. Gramegna, and S. P. Kulik, EPL (Europhysics Letters) 87, 64003 (2009)

[10] B. Bessire, C. Bernhard, T. Feurer, and A. Stefanov, New Journal of Physics 16, 033017 (2014).

[11] L. Olislager, E. Woodhead, K. Phan Huy, J.-m. Merolla, P. Emplit, and S. Massar, Physical Review A 89, 052323 (2014)

[12] H. C. Lim, A. Yoshizawa, H. Tsuchida, and K. Kikuchi, Optics express 16, 22099 (2008).

[13] H. C. Lim, A. Yoshizawa, H. Tsuchida, and K. Kikuchi, European Conference on Optical Communication, ECOC 16, 16052 (2008)

[14] T. E. Chapuran, P. Toliver, N. a. Peters, J. Jackel, M. S. Goodman, R. J. Runser, S. R. McNown, N. Dallmann, R. J. Hughes, K. P. McCabe, J. E. Nordholt, C. G. Peterson, K. T. Tyagi, L. Mercer, and H. Dardy, New Journal of Physics 11 (2009), 10.1088/1367-2630/11/10/105001

[15] I. Herbauts and B. Blauensteiner, Optics Express 21, 29013 (2013), arXiv:arXiv:1307.5462v1.

[16] J. M. Donohue, J. Lavoie, and K. J. Resch, Physical Review Letters 113, 163602 (2014), arXiv:1410.4524.

[17] A. Pe'er, B. Dayan, A. a. Friesem, and Y. Silberberg, Physical Review Letters 94, 1 (2005), arXiv:0411146 [quant-ph].

[18] A. PeYer, Y. Silberberg, B. Dayan, and A. Friesem, Phys. Rev. A 74, 1 (2006).

[19] K. a. O'Donnell and A. B. U'Ren, Optics letters 32, 817 (2007), arXiv:0701105 [quant-ph]

[20] Y. Shaked, R. Pomerantz, R. Z. Vered, and A. Pe?er, New Journal of Physics 16, 053012 (2014) arXiv:1209.4194.

[21] N. S. Bisht and R. Shimizu, J. Opt. Soc. Am. B 32, 550 (2015)

[22] E. Dauler, G. Jaeger, A. Muller, A. Migdall, and A. Sergienko, Journal Of Research Of The National Institute Of Standards And Technology 104, 1 (1999)
[23] K. G. Katamadze, N. A. Borshchevskaya, I. V. Dyakonov, A. V. Paterova, and S. P. Kulik, Laser Physics Letters 10, 045203 (2013).

24] S. Carrasco, M. B. Nasr, A. V. Sergienko, B. E. a. Saleh, M. C. Teich, J. P. Torres, and L. Torner, Optics letters 31, 253 (2006).

[25] S. Carrasco, A. Sergienko, B. Saleh, M. Teich, J. Torres, and L. Torner, Physical Review A 73, 1 (2006).

[26] S. Carrasco, J. Torres, L. Torner, A. Sergienko, B. Saleh, and M. Teich, Physical Review A 70, 043817 (2004)

[27] S.-Y. Baek and Y.-H. Kim, Physical Review A 80, 033814 (2009).

[28] M. Nasr, G. Giuseppe, B. Saleh, A. Sergienko, and M. Teich, Optics Communications 246, 521 (2005).

[29] M. Hendrych, X. Shi, A. Valencia, and J. Torres, Physical Review A 79, 023817 (2009).

[30] M. Okano, R. Okamoto, A. Tanaka, S. Subashchandran, and S. Takeuchi, Optics Express 20, 11 (2012).

[31] M. B. Nasr, S. Carrasco, B. E. A. Saleh, A. V. Sergienko, M. C. Teich, J. P. Torres, L. Torner, D. S. Hum, and M. M. Fejer, Physical Review Letters 100, 183601 (2008).

[32] D. A. Kalashnikov, K. G. Katamadze, and S. P. Kulik, JETP Letters 89, 224 (2009).

[33] K. G. Katamadze, A. V. Paterova, E. G. Yakimova, K. A. Balygin, and S. P. Kulik, JETP Letters 94, 262 (2011).

[34] K. G. Katamadze and S. P. Kulik, Journal of Experimental and Theoretical Physics 112, 20 (2011)

[35] A. Gatti, E. Brambilla, L. Caspani, O. Jedrkiewicz, and L. Lugiato, Physical Review Letters 102, 223601 (2009).

[36] F. A. Bovino, P. Varisco, A. Maria Colla, G. Castagnoli, G. Di Giuseppe, and A. V. Sergienko, Optics Communications 227, 343 (2003).

[37] C. Kurtsiefer, M. Oberparleiter, and H. Weinfurter, Physical Review A 64, 023802 (2001).

[38] A. Ling, A. Lamas-Linares, and C. Kurtsiefer, Physical Review A 77, 043834 (2008)

[39] D. Ljunggren and M. Tengner, Physical Review A 72, 062301 (2005)

[40] L. Cardoso and G. Figueira, Optics Express 12, 3108 (2004)

[41] L. Cardoso and G. Figueira, Optics Communications 251, 405 (2005).

[42] A. P. Piskarskas, A. P. Stabinis, and V. Pyragaite, IEEE Journal of Quantum Electronics 46, 1031 (2010).

[43] A. Stabinis and J. Krupic, Optics Communications 271, 564 (2007)

[44] K. Yamane, T. Tanigawa, T. Sekikawa, and M. Yamashita, Optics Express 16, 18345 (2008)

[45] G. Brida, M. Chekhova, I. Degiovanni, M. Genovese, G. Kitaeva, A. Meda, and O. Shumilkina, Physical Review Letters 103, 193602 (2009) 
[46] B. Dayan, A. Pe'er, A. Friesem, and Y. Silberberg, Physical Review Letters 94, 2 (2005)

[47] Y.-H. Kim, S. P. Kulik, and Y. Shih, Physical Review A 62, $011802(2000)$. 